\title{
Reproducción inducida de Pimelodus pictus con extracto de hipófisis de carpa (EHC) y Ovaprim ${ }^{\circledR}$
}

\section{Induced spawning of Pimelodus pictus with carp hypophysis extract (CHE) and Ovaprim ${ }^{\circledR}$}

\author{
Elizabeth Aya B, M.Sc, José Arias C, Ph.D. \\ Universidad de los Llanos, Instituto de Acuicultura de los Llanos (IALL), km7 vía Pto López, Villavicencio \\ (Meta) Colombia. Correspondencia: elizaya27@yahoo.com
}

Recibido: Septiembre de 2009; Aceptado: Julio de 2010.

\section{RESUMEN}

Objetivo. Comparar la respuesta a la maduración final de las ova y la tasa de ovulación usando dos tratamientos hormonales en hembras de Pimelodus pictus. Materiales y métodos. Hembras maduras recién capturadas fueron superestimuladas con Extracto de Hipófisis de Carpa (EHC) y mezcla del análogo de la hormona liberadora de la gonadotropina de salmón más domperidona, Ovaprim ${ }^{\circledR}$. Se utilizaron dosis totales de 5.5 y $7.7 \mathrm{mg} / \mathrm{kg}$, y 0.5 y $1 \mathrm{ml} / \mathrm{kg}$ respectivamente, dividida en dos dosis, e inyectadas con un intervalo de $12 \mathrm{~h}$ ( $10 \%$ de la dosis total en la primera inyección y $90 \%$ en la segunda). Resultados. Con tiempo de latencia de $6 \mathrm{~h} 30 \pm 20 \mathrm{~min}$ a $27.4 \pm 0.2^{\circ} \mathrm{C}$. Las hembras en todos los tratamientos respondieron por sobre el $50 \%$, siendo mayor la respuesta en ambos protocolos para EHC $(p<0.05)$. El diámetro ovocitario durante y después de los tratamientos aumentó en todos los casos, siendo mayor y diferente $(p<0.05)$, para los tratamientos con Ovaprim ${ }^{\circledR}$. La fecundidad se registró entre 818 a 1185 óvulos/hembra. Conclusiones. Se demuestra las posibilidades de reproducción inducida con dos inductores hormonales, siendo EHC superior a Ovaprim $®$.

Palabras clave: Reproducción, ovulación inducida, hormonas, Pimelodus pictus. (Fuentes: DeCS, AIMS) 


\section{ABSTRACT}

Objective. To induce spawning on Pimelodus pictus and to evaluate the response on total ova recovered maturation and ovulation rate using two hormonal treatments. Materials and methods. Mature females were captured and immediately induced with Carp Pituitary Extract (CPE) and a mixture of an analogue of salmon Gonadotropin-releasing hormone plus domperidone (Ovaprim $®$ ). A total doses of 5.5 and $7.7 \mathrm{mg} / \mathrm{kg}$ and 0.5 and $1 \mathrm{ml} / \mathrm{kg}$, were used. The total dose was divided into two doses and injected with an interval of $12 \mathrm{~h}$ (first dose: $10 \%$ and second dose: $90 \%$ respectively). Results. Females from all treatments responded with greater than $50 \%$, to a latent time of $6 \mathrm{~h} 30 \pm 20 \mathrm{~min}$ at $27.4 \pm 0.2^{\circ} \mathrm{C}$, the response being higher for both protocols with CPE $(p<0.05)$. The oocyte diameter before, during, and after all treatments increased, being higher and different $(p<0.05)$ for the treatments with Ovaprim $\AA$. Fecundity reproductive ranged from 818 to 1185 ova/female, with this variability being further discussed. Conclusions. This study demonstrates the potential of induced breeding with Pimelodus pictus as host for two hormonal inducers with the primary option being the use of CPE.

Key words: Reproduction, induced ovulation, hormones, Pimelodus pictus. (Sources: DeCS, AIMS)

\section{INTRODUCCIÓN}

El vistoso Pimelodus pictus, conocido como tigrito o pictus, es un pequeño bagre ornamental del sur de Colombia de gran demanda en los mercados internacionales, que representó el $5 \%$ del volumen total de peces exportados por Colombia en 2007 (1). Es un pez reofílico que se reproduce en la naturaleza en aguas subiendo entre abril y mayo de cada año (2-4), pero que no se reproduce naturalmente en confinamiento por lo que es necesario inducirlo con hormonas sexuales, siendo pocos los registros conocidos de respuestas reproductivas exitosas $(5,6)$, atribuyéndose gran parte de las dificultades para reproducírsele en confinamiento a la falta de información precisa sobre la respuesta a los diferentes inductores y sus protocolos de uso.

En esta especie se ha utilizado Extracto de Hipófisis de Carpa (EHC) (5-10), análogo de la hormona gonadotrópica corionica humana (Prymogonilß) $(6,8,9)$ y análogo de la GnRH de salmón más domperidona como antagonista de dopamina (Ovaprim $®$ ) (7), con resultados que han alcanzando solo un $10 \%$ de éxito en los procesos de maduración final ovocitaria, ovulación y desove $(5,6)$.
Con el objeto de aproximar a los cambios que se pudieran producir como consecuencia de la aplicación de las dos hormonas más utilizadas en la actualidad para la inducción de bagres neotropicales (EHC y Ovaprim $\AA$ ), se realizó el presente estudio para precisar la respuesta de la especie a los protocolos inductivos experimentados, el aumento del diámetro de los ovocitos como evidencia de la maduración final ovocitaria, el tiempo de latencia para la ovulación y la fecundidad reproductiva.

\section{MATERIALES Y MÉTODOS}

\section{Sitio deestudioy captura de ejemplares.}

Los parentales experimentados fueron capturados con atarraya de ojo de malla de $1 \mathrm{~cm}$ en aguas del río Ariari ( $3^{\circ} 28^{\prime} 53.28^{\prime \prime}$ N, $73^{\circ} 43^{\prime} 20.88^{\prime \prime}$ W) en alrededores de la localidad de Puerto Caldas, Meta, en el periodo de aguas subiendo durante los meses de abril y mayo de 2008.

$\begin{aligned} & \text { Número de capturas, manejo y } \\ & \text { preselección de ejemplares. De }\end{aligned}$
trescientos ejemplares capturados, se
preseleccionaron in situ 60 hembras
y 40 machos por sus características


externas de madurez reproductiva (11), e inmediatamente transportados a la unidad de peces ornamentales del Instituto de Acuicultura de la Universidad de los Llanos en la ciudad de Villavicencio (4 ${ }^{\circ}$ $05^{\prime} \mathrm{N} ; 73^{\circ} 37^{\prime} \mathrm{O}$ ), donde fueron recibidos y mantenidos en acuarios de $40 \mathrm{~L}$ con aguas preparadas al $2 \%$ de sal industrial (Sal UPIN $98 \%$ ) y temperatura $27.5 \pm$ $0.2^{\circ} \mathrm{C}$, oxígeno disuelto $5.3 \pm 0.2 \mathrm{mg} / \mathrm{L}$, $\mathrm{pH} 6.8 \pm 0.3$, conductividad eléctrica 150 $\pm 12 \mu \mathrm{S} / \mathrm{cm}$ (medidos con YSI $50000 \AA$ multiparametros) y dureza total $53 \pm 7 \mathrm{mg}$ / L (medida con kit water hardness Sera ${ }^{\circledR}$ ).

Posteriormente, previa tranquilización con MS-222 $®(0.5 \mathrm{mg} / \mathrm{L}), 45$ hembras con peso de $12.3 \pm 0.1 \mathrm{~g}$, tomado con balanza digital Ohaus $\AA$ con $0.01 \mathrm{~g}$ aproximación, fueron seleccionadas mediante medición de los diámetros de los ovocitos con reglilla adaptada a estéreomicroscopio y porcentualización de los estados de migración nuclear de los mismos, en una muestras de $30 \pm 2$ ovocitos obtenidos mediante suave estrujamiento, recibidos en suero fisiológico (solución estéril 0.9\% de $\mathrm{NaCl}$ ) y aclarados con solución Serra ( $85 \%$ etanol, $15 \%$ ácido acético y $5 \%$ formol) $(12,13)$.

\section{Selección de reproductoras y tratamientos. Las reproductoras} seleccionadas fueron aquellas que registraron ovocitos con diámetros de $820.8 \pm 12 \mu \mathrm{m}$ y $59.8 \pm 2.6 \%$ de los núcleos migrando. Las hembras seleccionadas fueron de inmediato y simultáneamente inducidas de manera aleatoria con los siguientes cuatro tratamientos, cada uno con nueve repeticiones: Tratamiento 1 (T1) con EHC, dosis total de $5.5 \mathrm{mg} / \mathrm{kg}$ de peso vivo (pv). Tratamiento 2 (T2) con EHC, dosis total $7.7 \mathrm{mg} / \mathrm{kg}$ pv. Tratamiento 3 (T3) con Ovaprim $\AA$, con dosis total de $0.5 \mathrm{~mL} / \mathrm{kg}$ de pv y Tratamiento 4 (T4) con Ovaprim $\AA$, con dosis total de $1 \mathrm{~mL} / \mathrm{kg}$ de pv. Para todos los tratamientos se utilizó como protocolo de aplicación una inyección inicial equivalente al $10 \%$ de la dosis total y 12 horas después una aplicación final equivalente al $90 \%$ de la dosis total. Seis hembras más seleccionadas fueron utilizadas como grupo control y recibieron $0.5 \mathrm{~mL} / \mathrm{kg}$ de suero fisiológico en la primera inyección y $1.0 \mathrm{~mL} / \mathrm{kg}$ en la segunda inyección. Los machos recibieron $5 \mathrm{mg} / \mathrm{kg} \mathrm{pv}$ de EHC en dosis única al momento de la primera inyección de las hembras.

Medición del diametro de ovocitos. Para determinar la variación del diámetro de los ovocitos por efecto del tratamiento, además de la muestra inicial descrita (DOPI: diámetro ovocitario preinducción), se realizaron dos muestras más/hembra así: diez minutos antes de aplicar la segunda dosis hormonal (DOSI: diámetro ovocitario antes de la segunda inyección hormonal), y una vez detectada la ovulación (DOPO: diámetro ovocitario pos ovulación).

Medición de la temperatura del agua. Después de aplicada la última inyección hormonal se registró la temperatura del agua cada hora con el propósito de establecer la temperatura media de respuesta de cada hembra/tratamiento y así el tiempo de latencia.

Determinación del momento de la ovulación. Después de la sexta hora de aplicada la última inyección hormonal las hembras de los diferentes tratamientos fueron revisadas cada 10 minutos para verificar el momento de la ovulación; ocurrida ésta y tomada la última muestra ovocitaria fue realizado el desove mediante estrujamiento y los óvulos recibidos en cajas de Petri. Para cada desove se contó manualmente el número de óvulos obtenidos y así la fecundidad reproductiva.

Análisis estadístico. Los datos fueron sometidos a análisis de varianza (ANOVA) y luego comparados con test de Tukey (14). La significancia estadística fue inferida a $p<0.05$. Los resultados fueron reportados como promedio \pm desviación estándar.

\section{RESULTADOS}

La tabla 1 registra los resultados de la respuesta reproductiva de las hembras a los diferentes tratamientos. Nótese la homogeneidad de los diferentes lotes conformados tanto en el peso corporal como 
Tabla 1. Respuesta ovulatoria, tiempo de latencia y fecundidad reproductiva de hembras de $P$. pictus, inducidas con dos diferentes dosis de EHC y Ovaprim ${ }^{\circledR}$.

\begin{tabular}{|c|c|c|c|c|c|}
\hline Tratamiento $^{1}$ & $\begin{array}{l}\text { Peso } \\
\text { corporal } \\
\text { (g) }\end{array}$ & $\begin{array}{c}\text { Migración } \\
\text { nuclear } \\
\text { preinducción \% }\end{array}$ & $R /{ }^{2}(\%)$ & $\begin{array}{c}\mathrm{TL}^{3} \text { horas } \\
\text { /temp }\end{array}$ & $\begin{array}{c}\text { Fecundidad } \\
\text { reproductiva }{ }^{4} \\
\text { óvulos/ hembra }\end{array}$ \\
\hline 1. EHC $5.5 \mathrm{mg} / \mathrm{kg}$ & $12.3 a \pm 0.2$ & $59.0 a \pm 2.8$ & $7 / 9(78) a$ & $\begin{array}{l}6.30 a \pm 0.18 \\
/ 27.1 a \pm 0.1\end{array}$ & $1185.2 \mathrm{a} \pm 323.3$ \\
\hline 2. EHC $7.7 \mathrm{mg} / \mathrm{kg}$ & $11.9 \mathrm{~b} \pm 0.1$ & $59.3 a \pm 3$ & $6 / 9(67) a b$ & $\begin{array}{c}6.36 a \pm 0.6 \\
/ 27.3 a \pm 0.2\end{array}$ & $1104.2 a \pm 193.8$ \\
\hline 3. Ovaprim $(0.5 \mathrm{~mL} / \mathrm{kg}$ & $12.5 a \pm 0,1$ & $60.2 \mathrm{a} \pm 2$ & $5 / 9(56) b$ & $\begin{array}{c}6.36 a \pm 0.6 \\
/ 27.6 a / \pm 0.1\end{array}$ & $938.7 b \pm 65.5$ \\
\hline 4. Ovaprim(B) $1 \mathrm{~mL} / \mathrm{kg}$ & $12.4 \mathrm{a} \pm 0.2$ & $60.3 a \pm 2$ & $5 / 9(56) b$ & $\begin{array}{l}6.42 b \pm 0.24 \\
/ 27.4 a \pm 0.1\end{array}$ & $817.8 b \pm 110.7$ \\
\hline Control $\mathrm{NaCl} 0.9 \%, 1 \mathrm{~mL} / \mathrm{kg}$ & $12.3 a \pm 0.1$ & $60.0 \mathrm{a} \pm 3$ & $0 / 6(0)$ & - & - \\
\hline
\end{tabular}

${ }^{1}$ Tratamiento; inductor, dosis total, protocolos de aplicación: 10\% en la primera inyección, intervalo de 12 horas y $90 \%$ en la segunda inyección. ${ }^{2} \mathrm{R} /=$ Respuesta ovulatoria=hembras con ovulación y huevos estrujados/ hembras inducidas (porcentaje). ${ }^{3} T L=T i e m p o$ de latencia=tiempo transcurrido desde la aplicación de la ultima inyección hormonal hasta el momento de la ovulación y estrujamiento. h/temp=horas, minutos/temperatura del agua. ${ }^{4}$ Fecundidad reproductiva=Número de óvulos estrujados. Entre filas, letras diferentes indican diferencias significativas $(p<0.05)$.

en las condiciones de madurez (migración ovocitaria preinducción), antes de los experimentos. En todos los tratamientos hubo respuestas ovulatorias del $56 \%$ o más, siendo los tratamientos con EHC los que mayores respuestas presentaron $(p<0.05)$. En cuanto al tiempo de latencia (tiempo transcurrido desde la aplicación de la ultima inyección hormonal hasta el momento de la ovulación y estrujamiento), fue similar para tres tratamientos, siendo diferente estadísticamente sólo para el tratamiento 4 con Ovaprim, obsérvese que la temperatura se mantuvo igual $(p<0.05)$ en todos los casos. Productivamente (fecundidad reproductiva), los tratamientos con EHC fueron mejores que los tratamientos con Ovaprim, pero sin mostrar diferencia estadística entre ellos. En el tratamiento control no se obtuvo respuesta ovulatoria.

La figura 1 muestra la variación del diámetro de los ovocitos durante los procedimientos. Se precisa que en este estudio se parte para todos los tratamientos de diámetros de $820.8 \pm 28.3 \mu \mathrm{m}$. Para el momento de la aplicación de la segunda dosis inductiva se encontró un aumento del diámetro ovocitario entre el 2.7 y el $5.1 \%$, sin diferencias estadísticas significativas entre tratamientos $(p<0.05)$, para todos los tratamientos excepto para el tratamiento control que disminuyó $1.8 \%$ el diámetro ovocitario. Para el momento de la ovulación los diámetros ovocitarios aumentaron aun más para todos los tratamientos inductivos siendo mayores en los tratamientos con Ovaprim ( $\mathrm{T} 3=988.9 \pm 24.9 \mu \mathrm{m}, 20.5 \%$ mayor que el diámetro inicial y diferente $[\mathrm{p}<0.05]$ a T4 $=934.3 \pm 24.9 \mu \mathrm{m}, 18.9 \%$ mayor al diámetro inicial), en tanto que los tratamientos con EHC aumentaron menos y sin diferencias estadísticas entre ellos $(\mathrm{T} 1=891.5 \pm 14.1 \mu \mathrm{m}, 8.6 \%$ mayor al

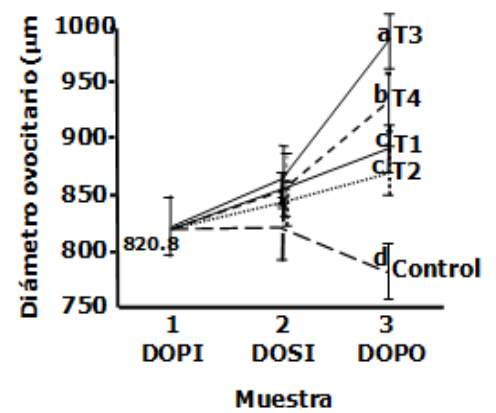

Figura 1. Efecto del tratamientos con EHC y Ovaprim $®$, sobre los diámetros ovocitarios $(\mu \mathrm{m})$, dehembras deP. pictus. $\mathrm{T} 1$, EHC $5.5 \mathrm{mg} / \mathrm{kg}, \mathrm{T} 2, \mathrm{EHC} 7.7 \mathrm{mg} / \mathrm{kg}$, T3, Ovaprim $®, 0.5 \mathrm{ml} / \mathrm{kg}$, T4, Ovaprim $1 \mathrm{ml} / \mathrm{kg}$. (1 DOPI=diámetro ovocitario pre inducción; 2 DOSI=diámetro ovocitario antes de la segunda inyección hormonal, 3 DOPO=diámetro ovocitario pos ovulación). Letras diferentes entre tratamientos indican diferencias significativas $(p<0.05)$. 
diámetro inicial y T2 $=869.7 \pm 25.1 \mu \mathrm{m}, 6$ $\%$ mayor al diámetro inicial). Los diámetros ovocitarios para el tratamiento control fueron al momento de la ovulación de los demás tratamientos inferiores y diferentes a los diámetros iníciales (T5 $=780.1 \pm$ $28.5 \mu \mathrm{m}, 9.5 \%$ inferior al diámetro inicial).

\section{DISCUSIÓN}

Pocas veces se ha reportado respuestas de maduración final y ovulación mayores del $50 \%$ en especie de peces utilizando diferentes hormonas, dosis y protocolos de inductores, como se encontró en este estudio, que resulta similar al comportamiento reportado para el bagre asiático del género Pangasius (15), pero contrario al registrado para otros bagres con respuestas positivas a los dos inductores experimentados. En tanto que en este trabajo se encontró que más hembras respondieron a los tratamientos con EHC que con Ovaprim $\AA$, para $P$. maculatus (16) y para Zúngaro jahu (17), encontraron que más hembras respondieron a tratamientos con Ovaprim.

Igual comportamiento al anterior se presentó para la fecundidad reproductiva, siendo esta mayor para los tratamientos con EHC, pudiéndose considerar este inductor como de primera opción para la especie $(6,7,9)$. De otra parte se trata de una fecundidad baja pero propia de la talla de las hembras (18) pero además muy irregular, lo que se podría explicar de una parte por la predisposición que parecen tener los silúridos a una gran variabilidad para este indicador (19) y con mayor frecuencia en especies del género Pimelodus $(20,21)$, y de otra, por lo dicho anteriormente en relación con las respuestas diferentes que los dos inductores propician.

El tiempo de latencia fue corto comparado con el tiempo de respuesta registrado en otros bagres bajo condiciones parecidas (16) y similar para todos los tratamientos $(p<0.05)$, esto último como ya fue anotado influenciado por la temperatura homogénea y semejante en la cual se desarrollaron los ensayos (12).
El peso de las hembras, los diámetros ovocitarios y el porcentaje medio de núcleos migrando antes de la primera inyección hormonal fueron similares para todos los grupos experimentados, ello hace muy confiables los resultados obtenidos en particular en cuanto a los aumentos de los diámetros ovocitarios durante los procesos inductores, condición varias veces requerida para el análisis de este evaluador $(22,23)$.

En todos los tratamientos los ovocitos aumentaron de tamaño en la medida en que las hembras fueron inyectadas, excepto en el tratamiento control, en donde por el contrario disminuyeron de diámetro. Pero resultó que el aumento de los diámetros de los ovocitos con respecto a las respuestas ovulatorias no fue el esperado, pues hubo menos respuestas en los tratamientos con Ovaprim, en donde el mayor aumento de diámetro de los ovocitos sugería mayor respuesta con ese inductor (24). No se tiene una explicación para este resultado, pero puede pensarse que se deba a una posible sobreestimulación ovocitaria por la mayor cantidad de hormona gonadotrópica recepcíonada por estos, como consecuencia del bloqueo de la dopamina por la domperidona que generaría una mayor concentración de hormonas gonadotrópicas y consecuentemente un mayor aumento del diámetro de los ovocitos $(22,24,25)$. Sin embargo se observó una aparente correlación inversa entre el diámetro ovocitario y la fecundidad reproductiva en los tratamientos con Ovaprim, contraria a los tratamientos con EHC, en los cuales se registró una mayor fecundidad reproductiva (mayor cantidad de óvulos estrujados) pero de menor diámetro, observaciones similares han sido registradas en otras especies en las que se ha explicado que ello se debe a que a mayor diámetro ovocitario menor es la cantidad de ovocitos que caben en el espacio abdominal y viceversa $(5,13,20)$.

Se puede concluir que hembras silvestres de Pimelodus pictus, sexualmente maduras, inducidas a la reproducción inmediatamente después de la captura con Extracto de Hipófisis de Carpa y Ovaprim $\AA$, responden a los dos inductores 
de manera diferencial, pudiéndose considerar la especie como receptora de los dos inductores hormonales probados en esta investigación. La respuesta al EHC fue mayor y más productiva por lo que se puede recomendar a este como el inductor de primera opción para la especie.

Se recomienda la realización de estudios de cría y manejo en cautiverio que permitan conseguir la madurez gonadal y subsecuente reproducción inducida y así generar el paquete tecnológico de la especie.

\section{Agradecimientos}

Al Instituto de Acuicultura de los Llanos (IALL), de la Universidad de los Llanos por el apoyo logístico.

\section{REFERENCIAS}

1. Mancera RNJ, Álvarez LR. Comercio de peces ornamentales en Colombia. Acta Biol Col 2008; 13:18.

2. Blanco CMC. Evaluación preliminar sobre la biología del "TIGRITO" Pimelodus pictus (Steindachner 1876), en el río Meta y resultados preliminares de algunos ensayos realizados en las instalaciones del vivero La Terraza sobre las especies Pimelodus pictus, (Steindachner: 1876) Megalamphodus sweglesi (Gery) y Cheirodon axelrodi (Schütz). Bogota: INDERENA; 1988.

3. Arias CJA. Ampliación del conocimiento de los peces de los llanos. Los peces ornamentales de la Orinoquía colombiana. Villavicencio. [Informe técnico]. Villavicencio, Colombia: Universidad de los Llanos; 1999.

4. Arias CJA, Pardo CSC. Apuntes sobre la Bioecología de cinco especies de peces ornamentales de la Orinoquía colombiana. Achagua 1999; 3(5):58-63.

5. Aya BE, Arias CJA. Reproducción inducida de Metynnis argenteus cf. y Pimelodus pictus con extracto de hipófisis de carpa. Villavicencio. [Informe técnico]. Villavicencio, colombia: Universidad de los Llanos; 2002.
6. Junca RV, Vallejo F. Inducción al desove en el tigrito Pimelodus pictus (Steindachner, 1876) mediante extractos de pituitaria de carpa y prymogonil@. [Tesis de grado]. Bogotá, Colombia: Universidad Jorge Tadeo Lozano; 1999.

7. Arias CJA, Aya BE. Los estudios de biología en ambientes naturales como estrategia para la producción ex situ de peces ornamentales de los Llanos Orientales de Colombia. Bogotá: INCODER, WWF; 2005.

8. Rodríguez RC, Estudio preliminar sobre la inducción a la madurez gonadal en cautiverio del tigrito Pimelodus pictus (Steindachnner, 1876). [Tesis de pregrado]. Bogotá, Colombia: Pontificia Universidad Javeriana; 1994.

9. Molano M. Estimulación a la maduración en cautiverio del tigrito Pimelodus pictus (Steindachner, 1876) (Pisces: Siluriformes) mediante extractos de pituitaria de carpa y Primogonyl. Bogota, Colombia: Universidad Jorge Tadeo Lozano; 1999.

10. Pardo CSC, Arias CJA, Aya BE, Gil FH. Experiencias preliminares de reproducción confinada en especies de peces ornamentales de la Orinoquía colombiana. Acuioriente 2000; (8):14-16. 
11. Woynarovich E, Horvath LA. Propagação artificial de peixes de águas tropicais. Manual de extensao. Brasilia, Brazil: FAO; 1983.

12. Vazzoler AC. Biología da reproduçâo de peixes teleósteos: Teoría e practica. Brazil: Maringá; 1996.

13. Gervásio LAF, Romagosa E, Borella MI, Batlouni SR. Induced spawning of hatchery-raised Brazilian catfish, cachara Pseudoplatystoma fasciatum (Linnaeu, 1760), Aquaculture 2004; 240:451-461.

14. Ayres M, Santos AS. Bioestat 2.0. Aplicações estatısticas nas áreas das ciências biológicas e médicas. 2000 .

15. Slembrouck J, Komarudin O, Maskur $\mathrm{H}$, Legendre M. Propagation of the Indonesian catfish, Indonésia: Pangasius; 2004.

16. Sampaio EV, Sato Y. Biologia reproductiva e desova induzida de duas espécies de bagres (Osteichthyes: Siluriformes) da bacia do rio São Francisco. Acta Scient Biol Scien 2006; 28(3):263-68

17. Martins DM. Reprodução induzida de jaú (Zungaro jahu) Anâlise das características seminais e ovocitárias. [Tesis de Maestría]. Minas Gerais-Brasil: Universidad Federal de Lavras; 2008.

18. Junca RV, Vallejo AF, Molano AM, Pinilla AG. Fecundidad en el tigrito Pimelodus pictus (Steindachnner, 1876). [Boletín científico]. Bogotá, Colombia: INPA; 2002.

19. Legendre $M$, Linhart $O$, Billard $R$. Spawning and management of gametes, fertilized eggs and embryos in Siluroidei. Aquat Living Resour 1996; 9:59-80.
20. Sato $Y N$, Fenerich-Verani JR, Verani $H$, Godinho P, Sampaio EV. Reproductive traits of yellow-mandi catfish Pimelodus maculatus Lacépède (Osteichthyes, Siluriformes) in captive breeding. Rev Bras Zool 1999; 16:981-86.

21. Pereira MB, Franco RS, Bizzotto MP, Volney $\mathrm{V}$, Pereira $\mathrm{GH}$. Reproductive activity and recruitment of the yellow-mandi Pimelodus maculatos (Teleostei:Pimelodidae) in the Igarapava Reservoir, Grande River. Brazil. Ichthyology 2007; 5(2):147-52.

22. Nun J, Dugué $R$, Corcuy $A N$, Duponchelle F, Renno JF, Raynaud $T$, et al. Induced breeding and larval rearing of Surubín, Pseudoplatystoma fasciatum (Linnaeus, 1766), from the Bolivia Amazon. Aquac Res 2008; 39:764-76.

23. García-Alonso J, Vizziano D. Induction of oocyte maturation in the white croaker Micropogonias furnieri (Pisces: Sciaenidae) by human chorionic gonadotropin. Braz J Biol 2004; 64(1):203-207.

24. Hill JE, Kilgore KH, Pouder DB. Survey of Ovaprim Use as a Spawning Aid in Ornamental Fishes in the United States as Administered through the University of Florida Tropical Aquaculture Laboratory. N Am J Aquaculture 2009; 71:206-209

25. Zohar Y, Mylonas CC. Endocrine manipulations of spawning in cultured fish: from hormones to genes. Aquaculture 2001; 197:99-136. 